\title{
Fever and skin redness in a 10-year-old boy
}

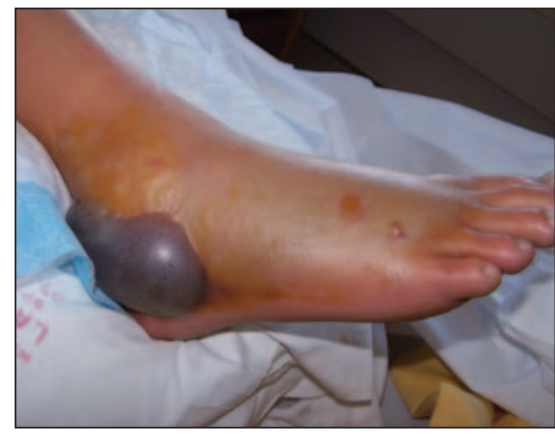

Figure 1: The right foot of the 10-yearold boy shortly after his cast was removed. Note the severe swelling, erythema and large bulla.

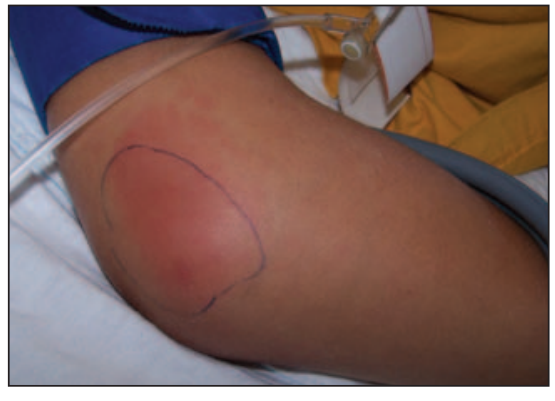

Figure 2: The patient's right elbow, which had swelling, erythema, pain and limited range of motion. The pen outline was drawn about 12 hours before this photograph was obtained.

\section{A} Io-year-old boy presented with a 2-day history of nausea, vomiting, malaise and fatigue as well as increasing pain, redness and swelling of his right foot and leg, right elbow and the fifth metacarpophalangeal joint of his right hand. Three days earlier the boy fell off a trampoline and fractured the cuboid and talus bones of his right ankle, which had been treated by casting. His right hand and elbow were not injured, and he had received only acetaminophen for analgesia. He had a 6-year history of dermatomyositis for which he was taking folic acid and methotrexate. The boy appeared ill, and he had a fever $\left(38.4^{\circ} \mathrm{C}\right)$.

After removing his cast, we found severe skin redness, warmth and swelling that extended from the tips of the toes of his right foot to the tuberosity of his tibia (Figure I). Large bullae were present. There was noteworthy tenderness with light palpation and pain with passive joint movements, and the dorsalis pedis pulse in his right foot was diminished. His right elbow (Figure 2) and right fifth metacarpophalangeal joint (Figure 3) were red, warm and swollen; however the related $\hat{\hbar}$ pulses were normal. The results of laboratory tests showed leukocytosis (3I [normal 4-I2] $\times 10^{9} / \mathrm{L}$ ), with 30.2 $\times 10^{9} / \mathrm{L}$ neutrophils and a $40 \%$ left shift, and an elevated erythrocyte sedimentation rate of $103 \mathrm{~mm} / \mathrm{h}$.

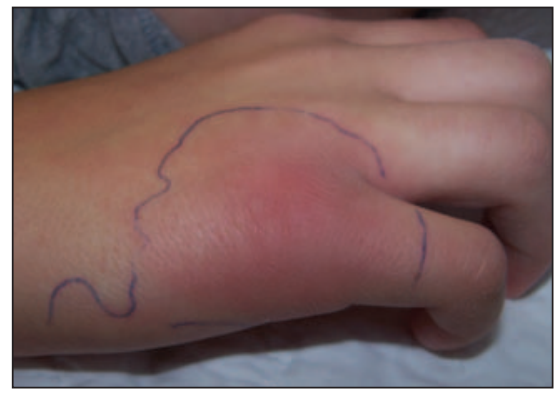

Figure 3: Fifth metacarpophalangeal joint of the boy's right hand, with erythema. The pen outline was drawn about 12 hours before this photograph was obtained.

\section{What is your diagnosis?}
A. Parvovirus Big infection
B. Acute rheumatic fever
C. Disseminated gonococcal infection
D. Polyarticular septic arthritis (non- gonococcal)
E. Reactive arthritis

\section{What is your next step?}

A. Obtain blood cultures and start broad-spectrum antibiotics

B. Perform viral serologic testing

C. Obtain an urgent consultation for possible surgical management

D. Both (a) and (c)

E. Initiate anti-inflammatory medications with observation in hospital

See page 1428 for diagnosis.

CMAJ invites contributions to the What is your call? column, which uses multiple-choice questions to guide a focused image-based discussion of the diagnosis or management of clinical cases. Submit manuscripts online at http://mc.manuscriptcentral.com/cmaj. 
WHAT IS YOUR CALL?

\section{Discussion}

The answer is (d) polyarticular septic arthritis (nongonococcal), and the best next step is (d) obtain blood cultures, start broad-spectrum antibiotics and obtain an urgent surgical consultation.

Our concerns about necrotizing fasciitis prompted an urgent plastic surgery consultation, and we started broad-spectrum antibiotics intravenously. Exploration in the operating room revealed no fascial necrosis in the right lower leg. The results of Gram staining of joint aspirates showed $4+$ pus ( $>4$ white blood cells per highpower field) and $4+$ gram-positive cocci (Io cocci per high-power field), and cultures from his ankle, elbow and metacarpophalangeal joints grew Streptococcus pyogenes. Blood cultures were negative for bacterial growth.

After several days of intravenous antibiotics his fever abated, he improved clinically and he was discharged. He received 6 additional weeks of intravenous and oral antibiotics because osteomyelitis could not definitively be ruled out based on follow-up imaging alone. A bone scan was not performed.

Monoarticular septic arthritis: Monoarticular septic arthritis is the most common form of infectious arthritis. Causative organisms are agedependent, but in children over the age of I month, Staphylococcus aureus, $S$. pyogenes and Streptococcus pneumonaie are most common. Depending on host and epidemiological factors, a variety of the bacterial, mycobacterial and fungal agents can be causative.

Polyarticular septic arthritis: Generally, less than $10 \%$ of septic arthritis is polyarticular. ${ }^{1}$ Causative agents of are typically Neisseria gonorrhoeae, Salmonella or $S$. aureus. S. pyogenes is rarely associated with polyarticular infection and is usually seen in patients with underlying joint disease or immunosuppression..$^{2,3}$ Occasionally, polyarticular arthritis can be mistaken for reactive arthritis. ${ }^{4}$ A 13-year review

Table 1: Selected differential diagnoses of polyarticular septic arthritis

\begin{tabular}{ll}
\hline Condition & \multicolumn{1}{c}{ Characteristics } \\
\hline $\begin{array}{l}\text { Parvovirus B19 } \\
\text { infection }\end{array}$ & $\begin{array}{l}\text { Symmetric polyarthritis or oligoarthritis; joint symptoms more } \\
\text { common among adults than among children }\end{array}$ \\
$\begin{array}{l}\text { Rheumatic fever } \\
\text { Migratory, large joint polyarthritis; pain is generally worse } \\
\text { than inflammation, follows Streptococcus pyogenes pharyngitis }\end{array}$ \\
$\begin{array}{l}\text { Disseminated } \\
\text { gonococcal infection } \\
\begin{array}{l}\text { Polyarticular septic } \\
\text { arthritis }\end{array}\end{array}$ & $\begin{array}{l}\text { Asymmetric, additive, purulent arthritis; can have associated } \\
\text { tenosynovitis. }\end{array}$ \\
Reactive arthritis & Asymmetric arthritis; prominent systemic symptoms \\
\hline
\end{tabular}

revealed 25 cases polyarticular septic arthritis, most commonly involving the knee or elbow, with $80 \%$ caused by S. aureus. ${ }^{5}$ Of these patients, about $50 \%$ had a history of underlying rheumatoid arthritis and $36 \%$ had immunosuppression because of a chronic illness. A higher mortality rate has been seen in cases with polyarticular compared with monoarticular arthritis (32\% v. $4 \%){ }^{5}$

In our case, the patient was immunosuppressed because of methotrexate therapy. Possible origins of his bacteremia include direct extension from cellulitis or arthritis of the ankle or, less likely, from the pharynx.

Differential diagnosis: In children, parvovirus BIg causes erythema infectiosum. Arthritis, symmetric and pauciarticular ( $\leq 4$ joints affected), often of the knees, rarely complicates pediatric infection (Table I). In adults, parvovirus Brg infection can present as an acute polyarthritis ( $\geq 5$ joints affected), with the proximal interphalangeal or metacarpophalangeal joints most often involved and about $75 \%$ of patients developing a rash.

Rheumatic fever is a nonsuppurative sequela of $S$. pyogenes pharyngitis and usually occurs $2-4$ weeks after infection. Arthritis is often the initial symptom. Migratory, large joint arthritis is I of the 5 major Jones criteria used for diagnosis. There is typically overlap in onset between different joints, and objective findings of inflammation are less common than joint pain.

Disseminated gonococcal infection occurs in more than $3 \%$ of patients with Neisseria gonorrhoeae. It presents with a spectrum of symptoms, ranging from a triad of tenosynovitis, dermatitis and polyarthritis to frank, purulent arthritis with no skin lesions. A history of sexual activity would be consistent with this infection. If gonococcal infection is present in a nonsexually active child, sexual abuse should be considered.

Reactive arthritis generally presents as a symmetric mono- or oligo-arthritis, which mainly affect the lower limbs. Joint symptoms appear days to weeks after a genitourinary or gastrointestinal infection, with no pathogen detected within the affected joint. Mucosal involvement (urethritis, stomatitis) and eye inflammation are common.

\section{Sergio T. Fanella MD}

Department of Medical Microbiology

Fred Y. Aoki MD

Department of Clinical Pharmacology

University of Manitoba

Winnipeg, Man.

Competing interests: None declared.

\section{REFERENCES}

I. Frank G, Mahoney HM, Eppes SC. Musculoskeletal infections in children. Pediatr Clin North Am 2005;52:1083-106.

2. Diaz-Borjon A, Lian S. Multifocal septic arthritis: an unusual complication of invasive Streptococcus pyogenes infection. Am JMed 2005; II 8:924-5.

3. Elwood RL, Pelszynski MM, Corman LI. Multifocal septic arthritis and osteomyelitis caused by group A Streptococcus in a patient receiving immunomodulating therapy with etanercept. Pediatr Infect Dis J 2003;22:286-8.

4. Christodoulou C, Gordon P, Coakley G. Polyarticular septic arthritis. BMJ 2006;333:1107-8.

5. Dubost JJ, Fis I, Denis P, et al. Polyarticular septic arthritis. Medicine (Baltimore) 1993;72:296-310. 